\title{
Identification of Circulating Diagnostic Biomarkers for Coronary Microvascular Disease in Postmenopausal Women Using Machine-Learning Techniques
}

\author{
Alicia Arredondo Eve 1,+D , Elif Tunc 1,2,+, Yu-Jeh Liu ${ }^{1}$, Saumya Agrawal ${ }^{3}$, Huriye Erbak Yilmaz ${ }^{2,4}$, \\ Sadık Volkan Emren ${ }^{2}$, Filiz Akyıldız Akçay ${ }^{2}$, , Luidmila Mainzer ${ }^{5,6}$, Justina Žurauskienè ${ }^{5,7,8}$ \\ and Zeynep Madak Erdogan 1,5,9,10,*(D)
}

Citation: Arredondo Eve, A.; Tunc, E.; Liu, Y.-J.; Agrawal, S.; Erbak Yilmaz, H.; Emren, S.V.; Akyıldız Akçay, F.; Mainzer, L.; Žurauskienè, J.; Madak Erdogan, Z. Identification of Circulating Diagnostic Biomarkers for Coronary Microvascular Disease in Postmenopausal Women Using Machine-Learning Techniques. Metabolites 2021, 11, 339. https:// doi.org/10.3390/metabo11060339

Academic Editor: Pollen K. Yeung

Received: 8 April 2021

Accepted: 20 May 2021

Published: 25 May 2021

Publisher's Note: MDPI stays neutra with regard to jurisdictional claims in published maps and institutional affiliations.

Copyright: (c) 2021 by the authors. Licensee MDPI, Basel, Switzerland. This article is an open access article distributed under the terms and conditions of the Creative Commons Attribution (CC BY) license (https:/ / creativecommons.org/licenses/by/ $4.0 /)$.
1 Department of Food Science and Human Nutrition, Division of Nutritional Sciences, University of Illinois at Urbana-Champaign, Urbana, IL 61801, USA; aliciaa2@illinois.edu (A.A.E.); mdeliftunc@gmail.com (E.T.); yliu233@illinois.edu (Y.-J.L.)

2 Research and Training Hospital, Katip Celebi University, Izmir 35620, Turkey; huriyeerbak@hotmail.com (H.E.Y.); vemren@hotmail.com (S.V.E.); drfilizkrd@hotmail.com (F.A.A.)

3 Department of Computer Science, University of Illinois, Urbana-Champaign, Urbana, IL 61801, USA; saumyaa2@illinois.edu

4 Izmir Biomedicine and Genome Center, Balcova, Izmir 35340, Turkey

5 Carl R. Woese Institute for Genomic Biology, University of Illinois at Urbana-Champaign, Urbana, IL 61801, USA; Imainzer@illinois.edu (L.M.); justina@illinois.edu (J.Ž.)

6 National Center for Supercomputing Applications, University of Illinois at Urbana-Champaign, Urbana, IL 61801, USA

7 Centre for Computational Biology, University of Birmingham, Birmingham B15 2T, UK

8 Institute of Cancer and Genomic Sciences, University of Birmingham, Birmingham B15 2TT, UK

9 Cancer Center at Illinois, University of Illinois, Urbana-Champaign, Urbana, IL 61801, USA

10 Beckman Institute for Advanced Science and Technology, University of Illinois at Urbana-Champaign, Urbana, IL 61801, USA

* Correspondence: zmadake2@illinois.edu

+ These authors contributed equally.

Abstract: Coronary microvascular disease (CMD) is a common form of heart disease in postmenopausal women. It is not due to plaque formation but dysfunction of microvessels that feed the heart muscle. The majority of the patients do not receive a proper diagnosis, are discharged prematurely and must go back to the hospital with persistent symptoms. Because of the lack of diagnostic biomarkers, in the current study, we focused on identifying novel circulating biomarkers of CMV (cytomegalovirus) that could potentially be used for developing a diagnostic test. We hypothesized that plasma metabolite composition is different for postmenopausal women with no heart disease, CAD (coronary artery disease), or CMD. A total of 70 postmenopausal women, 26 healthy individuals, 23 individuals with CMD and 21 individuals with CAD were recruited. Their full health screening and tests were completed. Basic cardiac examination, including detailed clinical history, additional disease and prescribed drugs, were noted. Electrocardiograph, transthoracic echocardiography and laboratory analysis were also obtained. Additionally, we performed full metabolite profiling of plasma samples from these individuals using gas chromatography-mass spectrometry (GC-MS) analysis, identified and classified circulating biomarkers using machine learning approaches. Stearic acid and ornithine levels were significantly higher in postmenopausal women with CMD. In contrast, valine levels were higher for women with CAD. Our research identified potential circulating plasma biomarkers of this debilitating heart disease in postmenopausal women, which will have a clinical impact on diagnostic test design in the future.

Keywords: metabolic-circulating biomarker; coronary microvascular dysfunction; postmenopausal women 


\section{Introduction}

Cardiovascular disease (CVD) is a major public health concern. It is the main cause of morbidity among women in the US [1]. Despite similar obesity prevalence in men and women, the risk of developing cardiovascular disease is greater in obese women (64\%) than in obese men (46\%) [2]. Additionally, women have a higher prevalence of coronary microvascular disease (CMD). In contrast, men tend to have atheroma and epicardial endothelial dysfunction or coronary artery disease [3]. The majority of female patients having CMD are postmenopausal women, and hormone-replacement therapies (HRT) decrease CMD risk up to $30 \%$ in this population, suggesting a role for estrogens in the development and progression of CMD [4].

CMD does not involve plaque formation, which blocks blood flow in the main coronary arteries. Instead, damage to the inner walls of blood vessels manifests via spasms and decreases blood flow to the heart muscle [5]. Patients complain about chest pain, shortness of breath, fatigue, and sleep problems. The current diagnosis strategy involves invasive and expansive methods, such as CFR, IMR or PET examination, which rules out the presence of CAD. Since there are no standard and specific diagnostic tests for CMD, patients do not receive a positive diagnosis when tested for coronary artery disease. Therefore, there is a clinical need for novel ways to diagnose, treat and prevent CMD in postmenopausal women.

Our objective was to identify novel markers of CMD in postmenopausal women. In this current study, our hypothesis is that postmenopausal women with CMD will have a distinct plasma metabolite profile compared to healthy women and women with CAD. Biomarkers we identified can be utilized in the clinic to diagnose women with CMD and significantly reduce future hospitalizations, cost and time for healthcare for diverse communities.

\section{Results}

Participants of the study were postmenopausal women with similar age and body mass indexes. Patients with angina or angina equivalent underwent nonischemic testing, in particular myocardial perfusion scintigraphy. Those who had severe epicardial coronary artery disease were included in CAD groups. Those who had normal or noncritical coronary stenosis with ischemia on myocardial perfusion imaging were included in the CMD group. Baseline characteristics are provided in Table 1. There was no statistically significant difference between the group averages of LDL, triglyceride, AST, ALT, urea, creatine, $\mathrm{Na}$, $\mathrm{K}$, glucose, $\mathrm{Hb}, \mathrm{WBC}$, PLT and MCV values. There was a significant difference between the values of total cholesterol ( $p$-value: 0.0072$)$ and HDL ( $p$-value: 0.0039$)$. The treatment protocols of the patients were compatible with existing co-morbid diseases and cardiac risk factors. Transthoracic echocardiography (systolic dysfunction, diastolic dysfunction, valve disorder, lv hypertrophy, pulmonary hypertension) and coronary angiography results of the patients are presented in Table 2. In the CAD group, 4 patients had more than one vessel atherosclerotic heart disease, 9 patients had single-vessel stents, 3 patients had 2 vessel stents, 3 patients had 3 vessel stents. 1 patient had undergone a coronary artery bypass graft operation. Coronary angiography of 22 patients in the CMD group was normal, and 1 patient had a single-vessel noncritical plaque. 
Table 1. Patient characteristics. $\mathrm{CMD}=$ coronary microvascular disease, $\mathrm{CAD}=$ coronary artery disease, $\mathrm{BMI}=$ body mass index, $\mathrm{COPD}=$ chronic obstructive pulmonary disease, $\mathrm{ACE}-\mathrm{ARB}$ diuretics = angiotensin-converting enzyme inhibitor, and angiotensin receptor blocker diuretics, $\mathrm{CA}=$ calcium channel blockers, $\mathrm{HDL}=$ high-density lipoprotein, $\mathrm{LDL}=$ low-density lipoprotein, AST $=$ aspartate aminotransferase, $\mathrm{ALT}=$ alanine aminotransferase, $\mathrm{Na}^{+}=$sodium, $\mathrm{K}^{+}=$potassium, $\mathrm{WBC}=$ white blood cell, $\mathrm{HB}=$ hemoglobin, $\mathrm{PLT}=$ platelet, $\mathrm{MCV}=$ mean corpuscular volume.

\begin{tabular}{|c|c|c|c|c|}
\hline Characteristics & Control $(n=26)$ & $\mathrm{CMD}(n=23)$ & CAD $(n=21)$ & $p$ Value $^{\mathrm{a}}$ \\
\hline \multicolumn{5}{|l|}{ Patient characteristics } \\
\hline Age, mean (SD), Y & $62(8)$ & $58(7)$ & $62(7)$ & 0.2057 \\
\hline BMI, median (IQR) ${ }^{b}$ & $31(30)$ & $30(30)$ & $30(29)$ & 0.8036 \\
\hline Hypertension, no. $(\%)$ & $17(65)$ & $8(34)$ & $11(52)$ & 0.101 \\
\hline Diabetes, no. $(\%)$ & $5(19)$ & $6(26)$ & $11(52)$ & 0.0412 \\
\hline Smoking, no. $(\%)$ & $2(7)$ & $0(0)$ & $1(4)$ & ND \\
\hline COPD or asthma, no. (\%) & $2(7)$ & $4(17)$ & $3(14)$ & ND \\
\hline HL, no. $(\%)$ & $5(19)$ & $5(21)$ & $5(23)$ & ND \\
\hline Rheumatology, no. (\%) & $0(0)$ & $1(4)$ & $1(4)$ & ND \\
\hline Thyroid, no. (\%) & $4(15)$ & $0(0)$ & $3(14)$ & ND \\
\hline \multicolumn{5}{|l|}{ Medication } \\
\hline Antithrombotic, no. (\%) & $6(23)$ & $14(60)$ & $20(95)$ & $<0.0001$ \\
\hline ACE-ARB diuretics, no. (\%) & $14(53)$ & $6(26)$ & $13(61)$ & 0.0408 \\
\hline CA channel blockers, no. (\%) & $8(30)$ & $3(13)$ & $6(28)$ & 0.3035 \\
\hline Beta blocker, no. $(\%)$ & $4(15)$ & $6(26)$ & $7(33)$ & 0.3507 \\
\hline Antianginal, no. $(\%)$ & $0(0)$ & $3(13)$ & $11(52)$ & $<0.0001$ \\
\hline Antihyperlipidemic, no. (\%) & $4(15)$ & $10(43)$ & $12(57)$ & 0.0097 \\
\hline \multicolumn{5}{|l|}{ Blood test results } \\
\hline Total cholesterol, mean (median), mg/dL & $237(234)$ & $213(202)$ & $192(185)$ & 0.0072 \\
\hline HDL, mean (median), mg/dL & $55(53)$ & $49(47)$ & $43(44)$ & 0.0039 \\
\hline LDL, mean (median), mg/dL & $150(147)$ & $128(126)$ & $113(99)$ & 0.0145 \\
\hline Triglyceride, mean (median), mg/dL & $162(146)$ & $172(141)$ & $179(151)$ & 0.815 \\
\hline Glucose, mean (median), mg/dL & $113(104)$ & $133(114)$ & $127(120)$ & 0.2305 \\
\hline Urea, mean (median), mg/dL & $14(13)$ & $13(13)$ & $14(13)$ & 0.5087 \\
\hline Creatinine, mean (median), mg/dL & $0.8(0.8)$ & $0.7(0.7)$ & $0.7(0.7)$ & 0.0491 \\
\hline AST, mean (median), U/L & $19(18)$ & $19(15)$ & $18(17)$ & 0.7581 \\
\hline ALT, mean (median), U/L & $18(17)$ & $17(16)$ & $20(17)$ & 0.5324 \\
\hline $\mathrm{Na}^{+}$, mean (median), meq/L & $140(140)$ & $139(139)$ & $140(139)$ & 0.6461 \\
\hline $\mathrm{K}^{+}$, mean (median), meq/L & $4.3(4.3)$ & $4.3(4.4)$ & $4.4(4.5)$ & 0.6139 \\
\hline WBC, mean (median), $\times 109 / \mathrm{L}$ & $7(7)$ & $7(7)$ & $8(8)$ & 0.3099 \\
\hline HB, mean (median), g/dL & $23(13)$ & $20(13)$ & $18(13)$ & 0.8631 \\
\hline PLT, mean (median) $\times 109 / \mathrm{L}$ & $283(270)$ & $275(254)$ & $307(303)$ & 0.4216 \\
\hline $\mathrm{MCV}$, mean (median), fL & $87(87)$ & $85(83)$ & $83(82)$ & 0.3882 \\
\hline
\end{tabular}

${ }^{\mathrm{a}} p$ value for 3-group comparison using one-way ANOVA multiple comparison and chi-squared analysis, ${ }^{\mathrm{b}}$ Calculated as weight in kilograms divided by height in meters squared.

Table 2. Characteristics of patients with CAD or CMD.

\begin{tabular}{cccc}
\hline Characteristics & Control $(\boldsymbol{n = 2 6 )}$ & CMD $(\boldsymbol{n}=\mathbf{2 3})$ & CAD $(\boldsymbol{n}=\mathbf{2 1})$ \\
\hline Transthoracic & & & \\
echocardiography & 1 & 1 & 0 \\
Systolic dysfunction & 15 & 11 & 13 \\
Diastolic dysfunction & 10 & 12 & 8 \\
Valve disorder & 4 & 1 & 0 \\
LV hypertrophy & 1 & 1 & \\
Pulmonary hypertension & & 22 & 4 \\
Coronary angiography & & 1 & \\
Normal & & & \\
Atherosclerotic heart disease & & & \\
\hline
\end{tabular}

To identify circulating biomarkers from plasma, we performed whole metabolite profiling using GC/MS analysis. The original dataset contained 175 metabolites. After data 
preprocessing and feature selection, we ended up with 45 metabolites. Using an unpaired t-test, we identified high stearic acid and ornithine as an indicator of CMD and valine as statistically different in the CAD group compared to the control group (Figure 1).
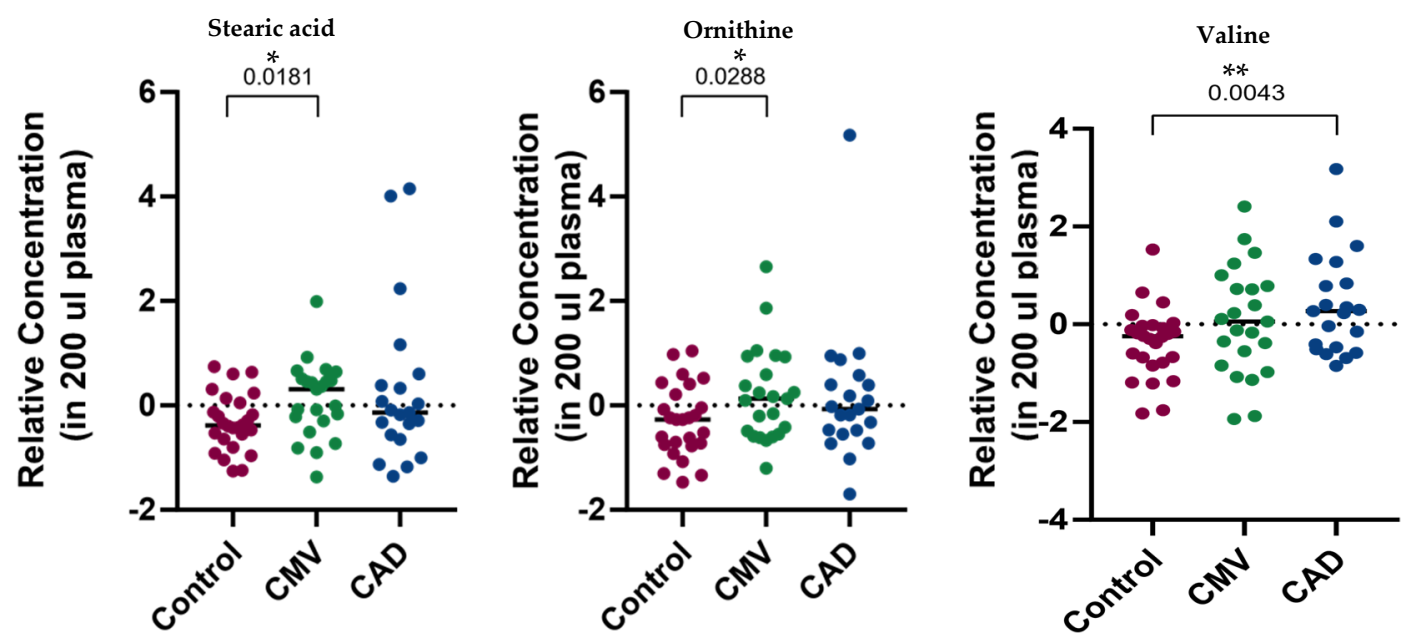

Figure 1. Significantly different metabolites between CMD vs. control and CAD vs. control groups, $\left(t\right.$-test: ${ }^{*} p<0.01$, ** $p<0.05)$.

To further develop a biomarker signature set, we used the RFECV algorithm, which iteratively computed the cross-validation score each time it eliminated a metabolite feature column (Figure S1). The classification achieved significantly better scores when more than 10 features were selected. The highest score was obtained when 15 metabolites were chosen (Figure S2). To test the classification performance of the identified signature, a binary classification was implemented using the random forest algorithm. The binary labels were "CMD" and "non-CMD", which combined both the CAD and control group samples. We tested the classification performance of stearic acid only, ornithine only, stearic acid and ornithine together, and the 15 metabolites set. The training and testing were done using 5 -fold cross-validation with a fixed random seed. The resulting performance was measured and visualized with receiver operating curves (ROC) and precision-recall (PR) curves. For the stearic acid, the mean area under the curve (AUC) was computed to be 0.58 with a standard deviation of 0.17 (Figure 2A, left panel). The mean F_1 score was computed to be 0.39 with a standard deviation of 0.13 (Figure 2A, right panel). The mean area under the curve (AUC) for ornithine was computed to be 0.55 with a standard deviation of 0.17 (Figure 2B, left panel). The mean F_1 score was computed to be 0.39 with a standard deviation of 0.14 (Figure 2B, right panel). Third, using the metabolite data from both stearic acid and ornithine, the mean area under the curve (AUC) was computed to be 0.60 with a standard deviation of 0.10 (Figure 2C, left panel). The mean F_1 score was computed to be 0.40 with a standard deviation of 0.14 (Figure 2C, right panel). Finally, using the metabolite data from the 15 metabolites selected by RFECV, the mean area under the curve (AUC) was computed to be 0.63 with a standard deviation of 0.04 (Figure 2D, left panel). The mean F_1 score was computed to be 0.40 with a standard deviation of 0.15 (Figure 2D, right panel). 
A

Stearic acid
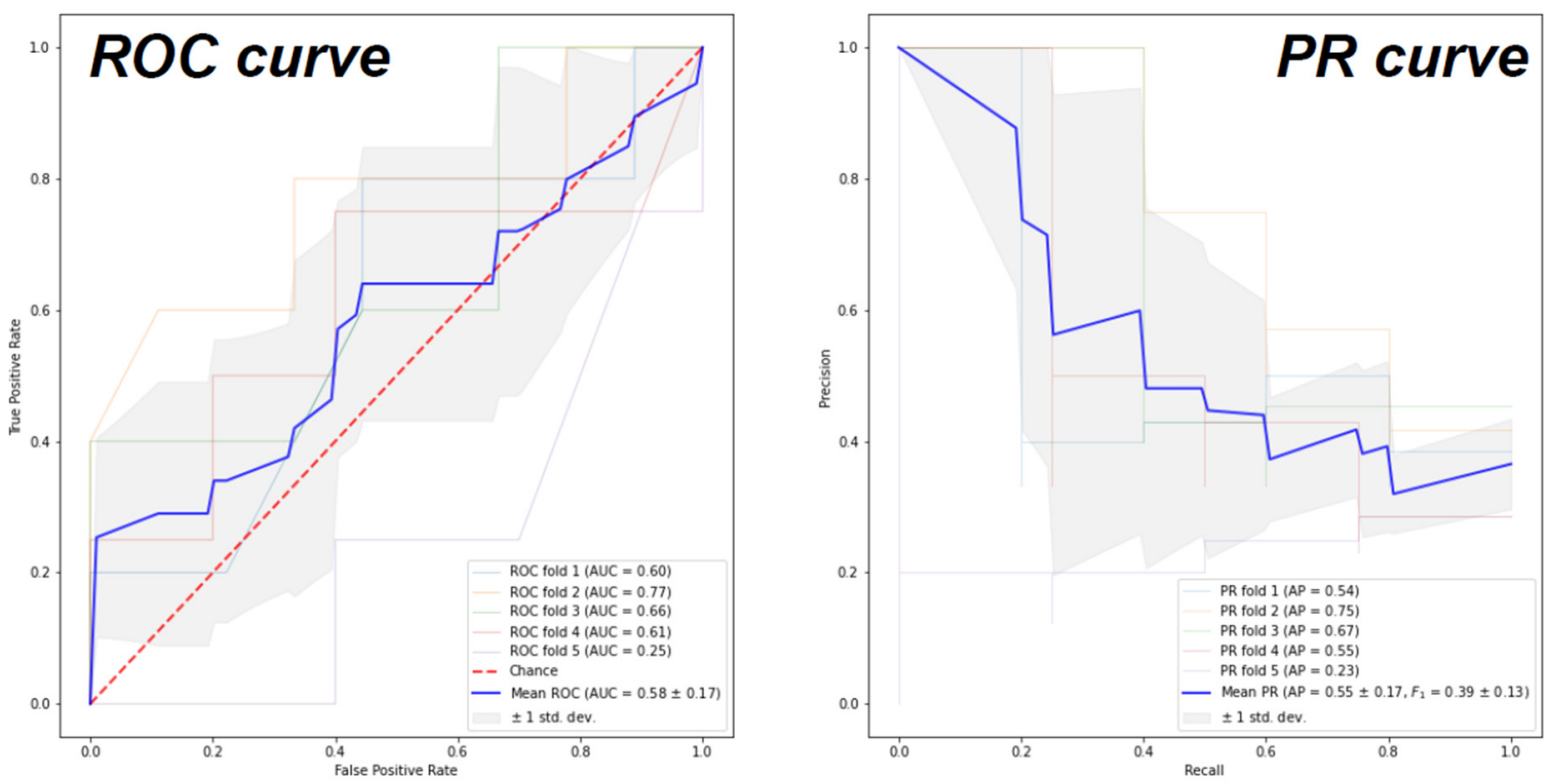

B

Ornithine
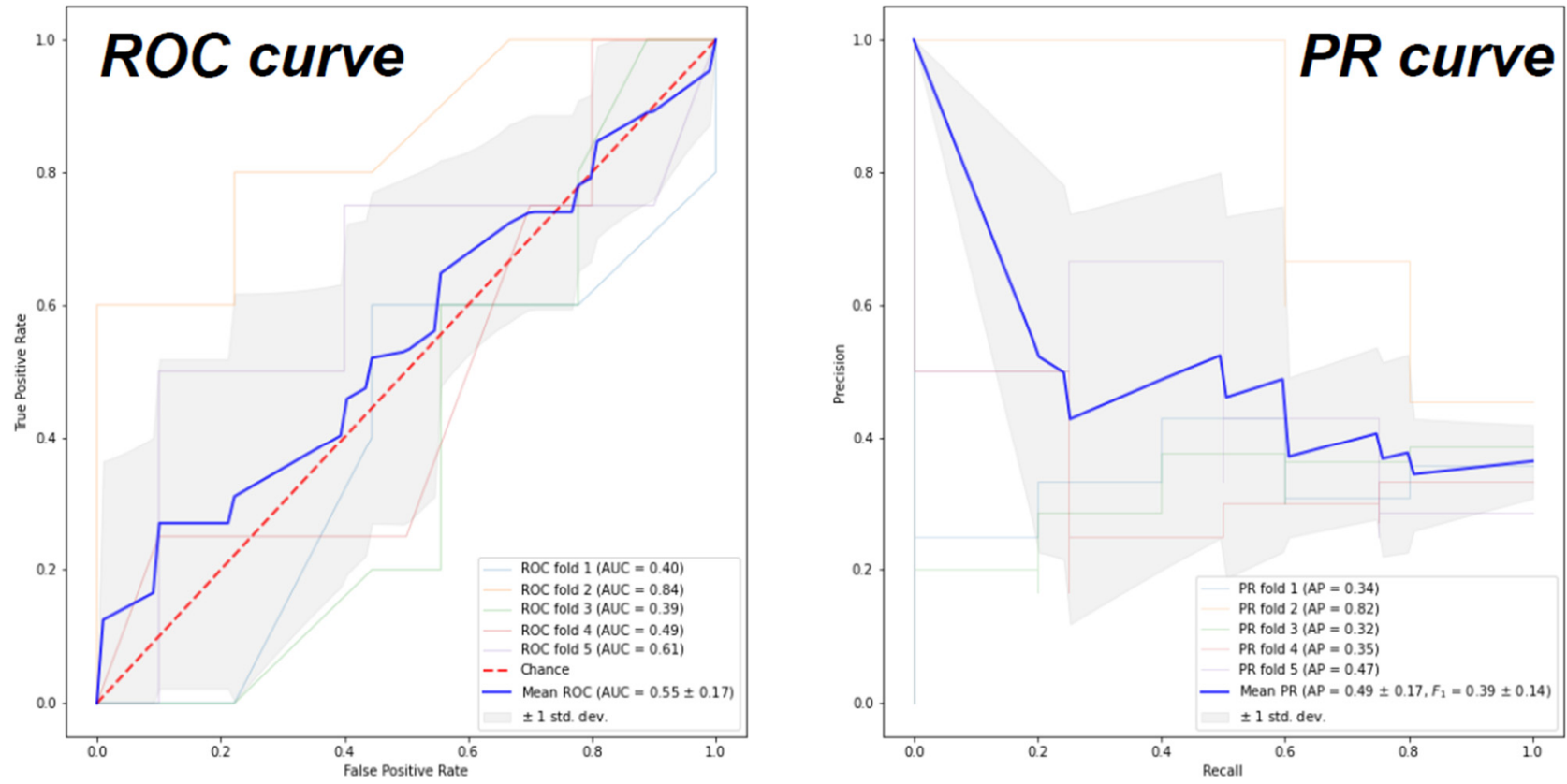

Figure 2. Cont. 
C

\section{Stearic acid + Ornithine}
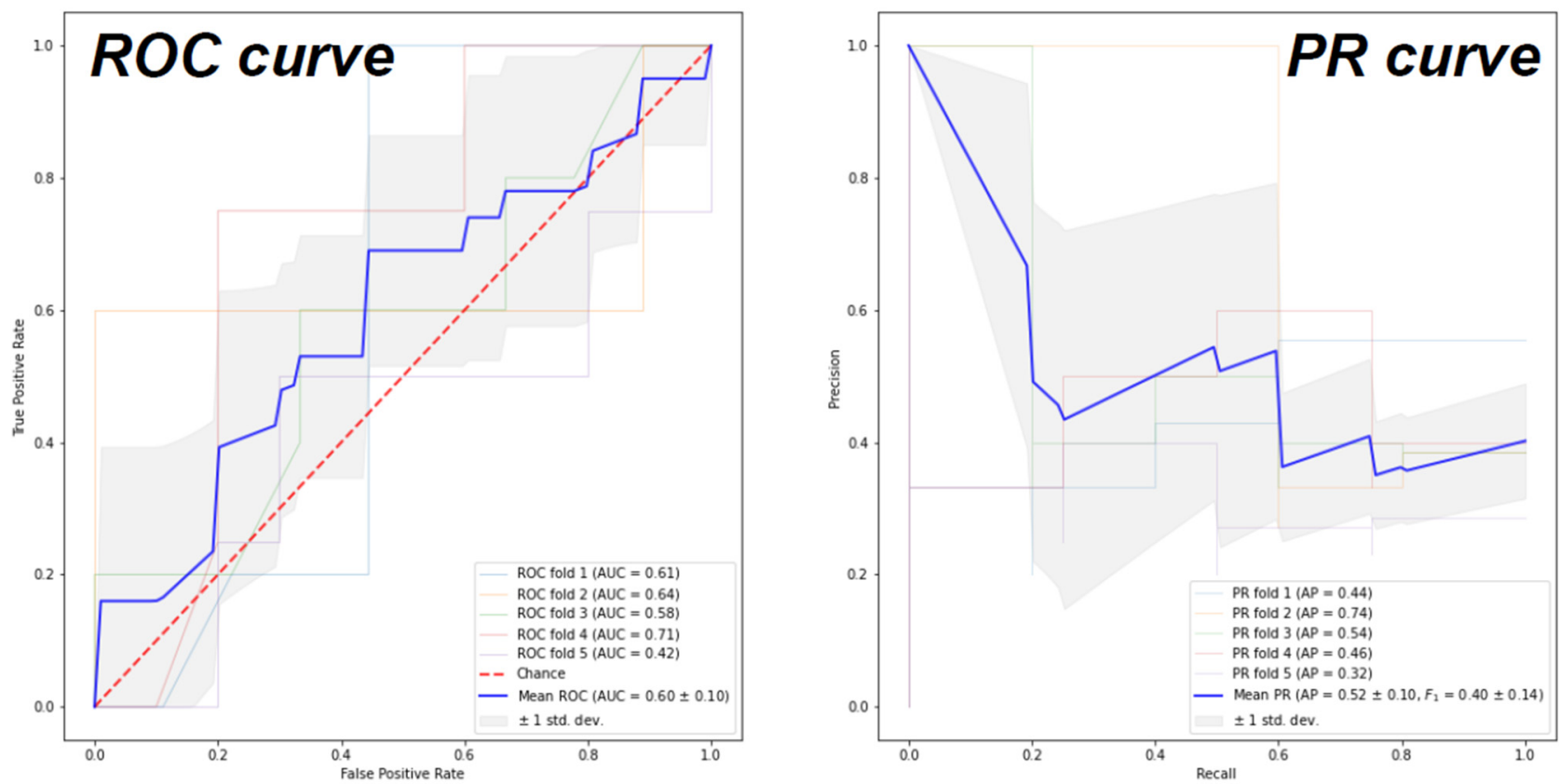

\section{5 metabolite signature}
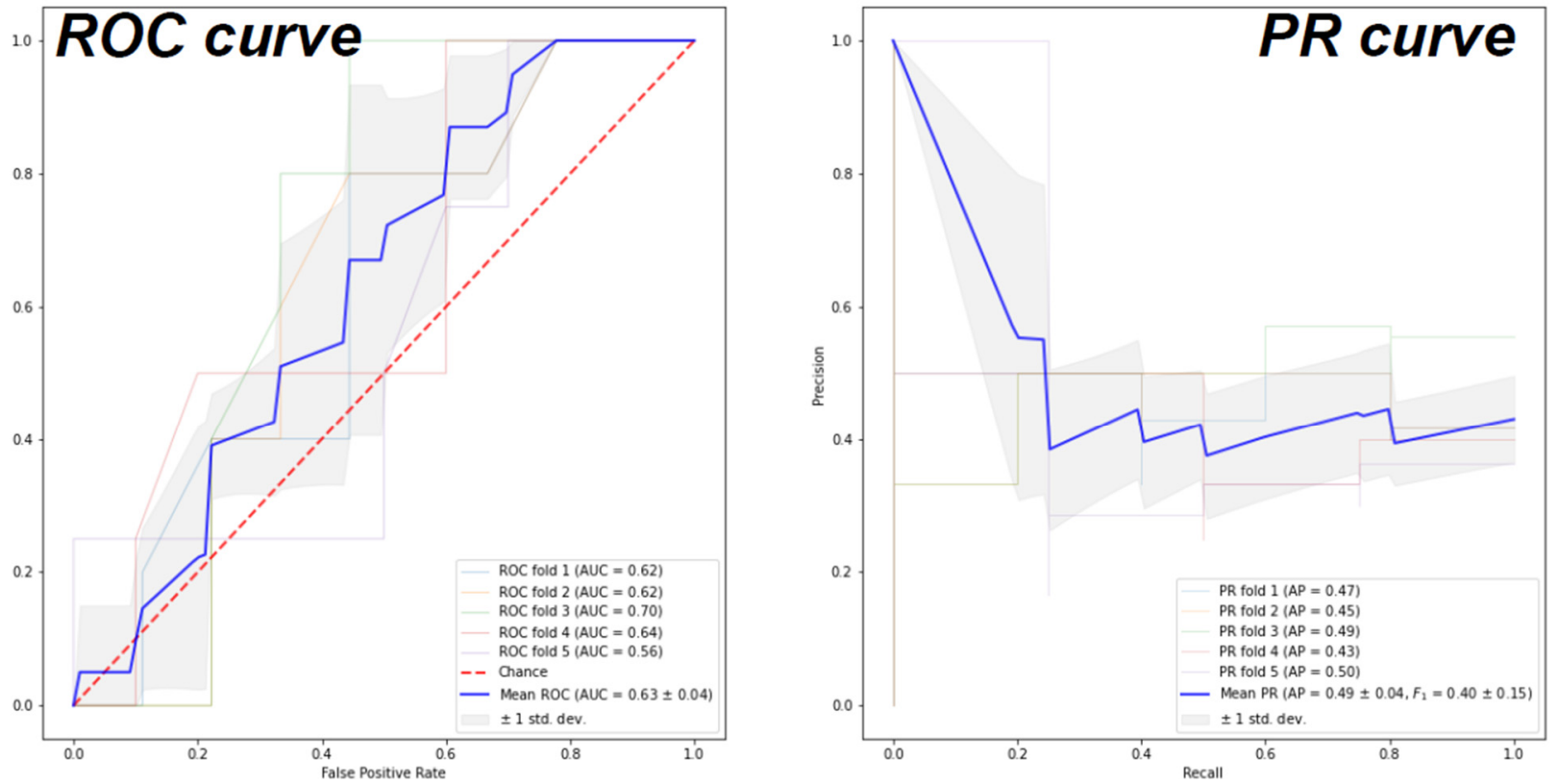

Figure 2. Classification performance validation using (A) stearic acid, (B) ornithine, (C) stearic acid + ornithine and (D) 15 metabolite signature.

\section{Discussion}

There are no specific diagnostic biomarkers for CMD. Because of the lack of specific diagnostic tests, many women who go to the emergency room with angina are discharged without a proper diagnosis, only to return to the hospital with more severe cardiac symptoms. We previously employed machine-learning techniques to identify biomarkers associated with other conditions, e.g., long-term-estrogen use [6], breast cancer risk [7], long-term broccoli consumption [8], and early predictors of liver carcinogenesis [9]. Feature selection and classification-based approaches enabled us to identify the best set of 
biological molecules that indicate health states with high specificity and selectivity. In the current study, we utilized plasma whole metabolite profiles and machine-learning algorithms to identify specific biomarkers of CMD. When we used classical statistical methods, which yielded stearic acid and ornithine as the metabolites that are statistically significant, they did not perform well to differentiate between CMD and non-CMD patients with high selectivity and specificity, hence lower mean AUC values. When we included 15 metabolites that were identified by the algorithm, our mean AUC increased, suggesting nonlinear methods may provide us a better set of biomarkers to develop as a diagnostic tool. However, plasma metabolites may not be ideal indicators of CMD, and current studies are underway to identify protein biomarkers, which we found to be better biomarkers for other pathological conditions before [7,10].

Long-chain acyl-carnitines, such as stearic acid, are formed from carnitine and acylCoAs by carnitine acyltransferases in mitochondria [11,12]. Because long-chain fatty Acids are the main energy substrates in the skeletal muscles and the heart, these tissues are considered essential contributors to the long-chain acyl-carnitine pool in plasma. The elevated levels of plasma acyl-carnitine have been linked to the progression of various diseases, including insulin resistance [13]; obesity, impaired glucose tolerance, type 2 diabetes [14-16]; and cardiovascular diseases [17-19]. In a cross-sectional study of 741 Chinese patients with T2DM, which aimed to estimate if there was an association between acyl-carnitine metabolites and CVD, investigators measured the fasting plasma levels of 25 acyl-carnitine metabolites in 288 individuals with CVD. Their analysis showed that some acyl-carnitine's were associated with the risk of CVD in T2DM [20]. In another case-cohort study (based on PREDIMED trial) with 980 individuals (229 cases and 751 non-cases), analysis of 3 acyl-carnitine groups (short-chain, medium-chain, and long-chain) found that increased short-chain and medium-chain plasma acyl-carnitines are associated with a higher risk of CVD independent of established CVD risk factors [21]. They suggested using short-chain acyl-carnitines as a potential biomarker of future stroke risk due to its increased concentration 3-4 years before the onset of CVD [21]. Consistent with their findings suggesting no association between stearic acid and CAD, in our study, we found an association between stearic acid and CMD, but not CAD. Long-chain saturated fatty acids, such as stearic acid consumed in significant amounts in the diet, can lead to metabolic disorder of glucose and lipids [22], but also can induce vascular endothelial inflammation, which has the potential to cause vascular dysfunction [23], ultimately causing diverse cardiovascular diseases. Some studies have found that consuming a Mediterranean diet could potentially decrease the cardiovascular risks that saturated fatty acids may yield [24,25].

In CMD vasospasm, vasoconstriction in smaller arteries of the heart is a major problem. In our study, we found ornithine plasma levels to be significantly higher in postmenopausal women with CMD. Ornithine is a metabolite, which is part of the pathway for NO production and arginine catabolism. Ornithine might be due to the increased activity of Arginase I enzyme, which is expressed in coronary microvasculature and can compete with nitric oxide synthase (NOS) for the use of L-arginine as a substrate [26], which results in the generation of ornithine and urea, instead of NO and L-citrulline. Obesity caused by a chronic high-fat diet plays a role in vascular endothelial cell dysfunction because of its low-grade, chronic inflammation [27], especially in the liver, where Arginase I is upregulated, contributing to changes in arginine metabolism [28]. In many studies, it is shown that obesity increases the expression of arginase I, which alters the bioavailability of arginine [29]. Arginine can be hydrolyzed to form urea and ornithine by arginase or can form NO by nitric oxide synthase. Both pathways compete for arginine [30]. Because of the increase in Arginase I activity, there is a reduced nitric oxide production, which is a major regulator of vascular homeostasis produced by endothelial cells, which play a role in maintaining normal vascular function by modulating vascular tone, inflammation and homeostasis [31].

CMD is a disease characterized by damage to the walls and inner lining of small coronary artery blood vessels due to constrictions and spasms of the heart wall. Addition- 
ally, decreased levels of estrogen, seen in postmenopausal women, are strongly correlated to coronary microvascular disease since estrogen modulates factors that are critical for the regulation of vascular relaxation. Estrogens improve nitric oxide function, which is a potent vasodilator [5]. One of the complications of T2DM is progression to cardiovascular disease (CMD), specifically due to microvascular dysfunction [32]. Arginase is believed to be an important regulator of NO bioavailability and endothelial function by hydrolyzing L-carnitine to ornithine and urea [31]. When Arginase activity is increased, it may reduce the production of $\mathrm{NO}$ and impair endothelial function, causing the microvascular disease to develop. In a prospective intervention study, which included 12 healthy individuals and 12 subjects with T2DM, plasma ratios of amino acids involved in arginase and NO synthase activities were determined [33]. It was found that individuals with T2DM had significantly higher levels of plasma ornithine than control when liquid chromatographytandem and mass spectrometry were done. In addition, T2DM patients had higher ratios of ornithine/citrulline and ornithine/arginine than the control group, indicating increased arginase activity. This study corroborates our finding, an association between increased levels of ornithine and CMD in postmenopausal women. Another case-control study analyzed plasma levels of different endogenous substrates for arginase in 298 patients, who were divided into 2 groups: Acute coronary syndrome (ACS) group, and stable angina (SAP) group [34]. Arginine, citrulline, ornithine, and methylated form of arginine c (SDMA) levels were measured using HPLC-MS/MS. Lower levels of arginine, citrulline and ornithine were found in both groups, as well as AMI (defined as the ratio of (arginine + citrulline + ornithine)/(ADMA + SMDA)). They concluded that AMI was an independent risk factor of acute coronary syndrome [34]. Findings from this study is consistent with ours, which did not find an association (no significant $p$ value) between ornithine and CAD.

We identified valine, a branched-chain amino acid, to be associated with CAD. Consistent with our findings, in a double-blinded study of 73 subjects, the biochemical profile of blood plasma in subjects with CAD and normal subjects (by angiography) was analyzed by high-resolution proton NMR spectroscopy [35]. It was found that high levels of lipids, alanine, and isoleucine/leucine/valine were observed in CAD subjects when compared to the control group. Valine, just like other amino acids, such as isoleucine, aspartate, and glutamate, provides the carbon skeleton for citric acid [36] and may play a role in the myocardial adaptation and mechanism adopted for restoring the ischemic injury of the heart [35], which serves as evidence that BBAA metabolism plays a role in cardiometabolic health [37-39]. In a women's health study that followed 27,041 healthy women for 18 years, isoleucine, leucine, and valine were found to be positively correlated with future CVD events [40]. Overall, our findings are consistent with these studies.

Combined with a western meat-rich diet, obesity can increase the levels of circulating BCAA (leucine, isoleucine and valine) to the point of causing non-alcoholic fatty liver disease and other related metabolic disorders by increasing the levels of free fatty acid (FFA) in plasma and in the liver, but also inhibiting the conversion of FFA into TG, causing FFA lipotoxicity in the ladder [41]. BCAA also can modulate glucose metabolism. It has been found that increase plasma concentration of BCAA is associated with the development of diabetes and insulin resistance [42].

In summary, we identified biologically relevant metabolites of microvessel function to be significantly different in postmenopausal women with CMD. Although classification performances of the identified molecules are not ideal, our studies provide proof of principle that plasma metabolite profiles can be used to develop diagnostic signatures for CMD. More future studies are underway to analyze inflammatory cytokines and other protein markers relevant to cardiovascular health. A combination of metabolomics and proteomics approaches combined with advanced machine learning techniques provides diagnostic biomarkers to be used in the clinic. 


\section{Materials and Methods}

\subsection{Study Design and Population}

Studies are approved by the Izmir Katip Celebi University Interventional Clinical Studies Institutional Review Board (IRB\#80). All research was carried out in compliance with the Helsinki Declaration. Donors provided broadly written consent for using their specimens in research. The consent document informed the donor that the donated specimens and medical data would be used for the general purpose of helping to determine biomarkers of CMD in postmenopausal women.

A total of 70 patients were included in this prospective observational cohort study. 23 of these patients were diagnosed with CMD (group 1), 21 patients were diagnosed with $\mathrm{CAD}$ (group 2), and 26 patients were defined as the control group (group 3). Inclusion criteria were as follows: having CAD-related chest pain, a positive non-invasive imaging result, and undergoing a successful coronary angiography with patients' consent. Exclusion criteria of the study were: being male or a premenopausal female, having a contraindication against coronary angiography. Patients who refused to participate in the study were also excluded from this research.

Anamnesis, physical examination, body mass index (BMI), medications, transthoracic echocardiogram, blood pressure measurements, and routine biochemical examinations (total cholesterol (TC), high-density lipoprotein (HDL) cholesterol, low-density lipoprotein (LDL) cholesterol, triglyceride, glucose, urea, creatinine, aspartate transaminase (AST), alanine transaminase (ALT), hemoglobin ( $\mathrm{Hb})$, white blood cell (WBC), mean corpuscular volume (MCV), platelet (PLT) levels) were recorded (Table 1).

Coronary angiography (CAG) procedure was applied to patients who underwent noninvasive imaging methods (cardiovascular stress test, myocardial perfusion scintigraphy) and was found to be positive. CAG was performed using the Judkins technique through the femoral or radial artery. Each coronary artery was displayed in at least two different plane images. All patients who accepted the procedure were informed about the study, and their written consent was obtained. After this procedure, a $5 \mathrm{cc}$ blood sample was obtained from patients who were found to have coronary artery disease or CMD as a result of coronary angiography. After obtaining the consent of patients, who were determined as the control group with similar demographic and medical characteristics, $5 \mathrm{cc}$ blood was taken. Venous blood samples were obtained from the antecubital vein. $100 \mu \mathrm{L}$ plasma samples prepared from blood samples were stored at $-80^{\circ} \mathrm{C}$ for processing.

\subsection{GC/MS Validation of the Metabolites by Whole Metabolite Profiling}

To detect and quantify the circulating metabolites in plasma, a gas chromatographymass spectrometry (GC/MS) analysis was performed at UIUC Metabolomics Center as described [7-10,43]. Briefly, $50 \mu \mathrm{L}$ of blood plasma was extracted using $1 \mathrm{~mL}$ of isopropanol:acetonitrile:water $(3: 3: 2, v / v)$ at $20{ }^{\circ} \mathrm{C}$ for $5 \mathrm{~min}$. After centrifugation, $0.5 \mathrm{~mL}$ of supernatant was dried in a SpeedVac concentrator and subsequently derivatized in two-steps: with $50 \mu \mathrm{L}$ methoxyamine hydrochloride (Sigma-Aldrich, St Louis, MO, USA) $\left(40 \mathrm{mg} / \mathrm{mL}\right.$ in pyridine) for $60 \mathrm{~min}$ at $50^{\circ} \mathrm{C}$, then with $50 \mu \mathrm{L}$ MSTFA $+1 \%$ TMCS (Thermo, Waltham, MA, USA) at $70{ }^{\circ} \mathrm{C}$ for $120 \mathrm{~min}$, followed by a 2-h incubation at room temperature. Hentriacontanoic acid ( $30 \mu \mathrm{L}$ of $1 \mathrm{mg} / \mathrm{mL}$ ) was added to each sample before derivatization for use as an internal standard for normalization. Metabolite profiles were acquired using a gas-chromatography mass-spectrometry (GC-MS) system (Agilent Inc, Santa Clara, CA, USA) consisting of an Agilent 7890 gas chromatograph, an Agilent 5975 MSD and 7683 B autosampler, as previously described [44]. Briefly, gas chromatography was performed on a ZB-5MS ( $60 \mathrm{~m} \times 0.32 \mathrm{~mm}$ ID and $0.25 \mathrm{~mm}$ film thickness) capillary column (Phenomenex, Torrance, CA, USA). The inlet and MS interface temperatures were $250{ }^{\circ} \mathrm{C}$, and the ion source temperature was adjusted to $230^{\circ} \mathrm{C}$. An aliquot of $1 \mathrm{~mL}$ was injected with a split ratio of 10:1. The helium carrier gas was kept at a constant flow rate of $2.4 \mathrm{~mL} / \mathrm{min}$. The temperature program was: $5 \mathrm{~min}$ isothermal heating at $70^{\circ} \mathrm{C}$, followed by an oven temperature increase of $5{ }^{\circ} \mathrm{C} / \mathrm{min}$ to ${ }^{\circ} \mathrm{C}$ after which a final $10 \mathrm{~min}$ incubation at $310^{\circ} \mathrm{C}$ was 
performed. The mass spectrometer was operated in positive electron impact mode (EI) at $69.9 \mathrm{eV}$ ionization energy at $m / z 30-800$ scan range. The scan range was set at least $50 \mathrm{~m} / z$ above the highest anticipated fragment. The minimum quality match for minor compounds was $\geq 80$ and for other peaks $\geq 90$. The spectra of all chromatogram peaks were evaluated using the AMDIS 2.71 (NIST, Gaithersburg, MD, USA) using a custom-built MS database (484 unique metabolites) [11]. Tentative substances were not reported. All known artificial peaks were identified and removed before data mining. To allow comparison between samples, all data were normalized to the internal standard in each chromatogram.

Differences in metabolite profiles among control, CAD and CMD were analyzed using Prism 7.0 (GraphPad software, San Diego, CA, USA, RRID:SCR_002798), after Zscores were calculated for each metabolite. An unpaired t-test was performed to identify metabolites that are different between each group. For clinical data, a one-way-ANOVA model and chi-squared analysis were fitted to test the statistical significance of differences between different groups, followed by Tukey's post hoc test. $p<0.05$ was considered significant.

\subsection{Machine Learning Analysis: Data Preprocessing, Feature Selection and Classification}

The raw data consisting of 175 metabolites measured across 70 patients was normalized using min/max scaling. Data imputation was then utilized to handle missing data and get the data ready for the classification task afterward. The metabolic feature columns, which had more than $40 \%$ of data missing, were eliminated. Next, we trained an iterative imputer on the rest of the data using the IterativeImputer class inside the Python sklearn.impute module to infill missing values. We iteratively tested the trained imputer by removing the data of one metabolite feature column and imputing the data afterward. At this step, the performance of the imputer was measured for each of the metabolites by observing the $\mathrm{R}^{2}$ (Figure S1). We used the $\mathrm{R}^{2}$ measure to eliminate the metabolite column with $R^{2}<0.3$ and had more than $5 \%$ of missing values across all patients. The last step of preprocessing was data standardization. We performed mean removal and variance scaling, which gave us zero mean and unit variance for each metabolite feature column. Preprocessing step reduced the feature columns to 75 metabolites, which were passed for further analysis. Feature selection was performed on the preprocessed dataset using the recursive feature elimination with cross-validation (RFECV) function inside the sklearn. Feature selection module. A random forest classifier was used as the estimator with 5-fold cv validation.

Supplementary Materials: The following are available online at https://www.mdpi.com/article/ 10.3390/metabo11060339/s1, Figure S1: RFECV algorithm results. RFEVC iteratively computed the cross-validation score each time it eliminated a metabolite feature column, Figure S2: Relative abundance of 15 metabolites in Control, CMD and CAD groups that gave the highest ROC in when random forest algorithm was used.

Author Contributions: Conceptualization, Z.M.E. and E.T.; methodology, A.A.E., E.T., H.E.Y., S.V.E., F.A.A.; software, Y.-J.L., L.M., J.Ž.; formal analysis, A.A.E., Y.-J.L., S.A.; resources, Z.M.E., E.T.; writing—original draft preparation, Z.M.E., E.T., A.A.E.; writing—review and editing, Z.M.E., E.T.; visualization, A.A.E., Y.-J.L.; supervision, Z.M.E.; project administration, Z.M.E.; funding acquisition, Z.M.E. All authors have read and agreed to the published version of the manuscript.

Funding: This work was supported by grants from the University of Illinois, ACES Future interdisciplinary research explorations grant (Z.M.E.), Office of International Programs-Conrad Award (Z.M.E.), National Institute of Food and Agriculture, U.S. Department of Agriculture, award ILLU698-331 (to Z.M.E.), National Center for Supercomputing Applications Faculty Fellows (to Z.M.E.) and TÜBİTAK 2219 Post Doctorate Research Scholarship Program-1059B191601914 (to E.T.).

Institutional Review Board Statement: Studies are approved by the Izmir Katip Celebi University Interventional Clinical Studies Institutional Review Board (IRB\#80). All research was carried out in compliance with the Helsinki Declaration. 
Informed Consent Statement: Donors provided broad written consent for using their specimens in research. The consent document informed the donor that the donated specimens and medical data would be used for the general purpose of helping to determine biomarkers of CMD in postmenopausal women.

Data Availability Statement: Data is available from authors upon request.

Acknowledgments: We would like to thank UIUC Metabolomics core, Lucas Li and Alex Ulanov.

Conflicts of Interest: Z.M.E. has an investigator-initiated grant from Karyopharm Therapeutics and is a co-inventor on several patents entitled "Novel Compounds Which Activate Estrogen Receptors and Compositions and Methods of Using the Same".

\section{References}

1. Mosca, L.; Barrett-Connor, E.; Wenger, N.K. Sex/gender differences in cardiovascular disease prevention: What a difference a decade makes. Circulation 2011, 124, 2145-2154. [CrossRef] [PubMed]

2. Wilson, P.W.; D'Agostino, R.B.; Sullivan, L.; Parise, H.; Kannel, W.B. Overweight and obesity as determinants of cardiovascular risk: The Framingham experience. Arch. Intern. Med. 2002, 162, 1867-1872. [CrossRef] [PubMed]

3. Sharaf, B.; Wood, T.; Shaw, L.; Johnson, B.D.; Kelsey, S.; Anderson, R.D.; Pepine, C.J.; Merz, C.N.B. Adverse outcomes among women presenting with signs and symptoms of ischemia and no obstructive coronary artery disease: Findings from the National Heart, Lung, and Blood Institute-sponsored Women's Ischemia Syndrome Evaluation (WISE) angiographic core laboratory. Am. Heart J. 2013, 166, 134-141. [CrossRef] [PubMed]

4. Alexandersen, P.; Tankó, L.B.; Bagger, Y.Z.; Qin, G.; Christiansen, C. The long-term impact of 2-3 years of hormone replacement therapy on cardiovascular mortality and atherosclerosis in healthy women. Climacteric 2006, 9, 108-118. [CrossRef] [PubMed]

5. Tunc, E.; Eve, A.A.; Madak-Erdogan, Z. Coronary Microvascular Dysfunction and Estrogen Receptor Signaling. Trends Endocrinol. Metab. 2020, 31, 228-238. [CrossRef] [PubMed]

6. Chen, K.L.A.; Liu, X.; Zhao, Y.C.; Hieronymi, K.; Rossi, G.; Auvil, L.S.; Welge, M.; Bushell, C.; Smith, R.L.; Carlson, K.E.; et al. Long-Term Administration of Conjugated Estrogen and Bazedoxifene Decreased Murine Fecal beta-Glucuronidase Activity Without Impacting Overall Microbiome Community. Sci. Rep. 2018, 8, 8166. [CrossRef] [PubMed]

7. Oktay, K.; Santaliz-Casiano, A.; Patel, M.; Marino, N.; Storniolo, A.M.V.; Torun, H.; Acar, B.; Madak Erdogan, Z. A Computational Statistics Approach to Evaluate Blood Biomarkers for Breast Cancer Risk Stratification. Horm. Cancer 2020, 11, 17-33. [CrossRef]

8. Eve, A.A.; Liu, X.; Wang, Y.; Miller, M.J.; Jeffery, E.H.; Madak-Erdogan, Z. Biomarkers of Broccoli Consumption: Implications for Glutathione Metabolism and Liver Health. Nutrients 2020, 12, 2514. [CrossRef]

9. Smith, B.P.; Auvil, L.S.; Welge, M.; Bushell, C.; Bhargava, R.; Elango, N.; Johnson, K.; Erdogan, Z.M. Identification of Early Liver Toxicity Gene Signatures Using Comparative Supervised Machine Learning. Sci. Rep. 2020, 10, 1-27. [CrossRef]

10. Madak-Erdogan, Z.; Band, S.; Zhao, Y.C.; Smith, B.P.; Kulkoyluoglu-Cotul, E.; Zuo, Q.; Casiano, A.S.; Wrobel, K.; Rossi, G.; Smith, R.L.; et al. Free Fatty Acids Rewire Cancer Metabolism in Obesity-Associated Breast Cancer via Estrogen Receptor and mTOR Signaling. Cancer Res. 2019, 79, 2494-2510. [CrossRef]

11. Kerner, J.; Hoppel, C. Fatty acid import into mitochondria. Biochim. Et Biophys. Acta (BBA) Mol. Cell Biol. Lipids 2000, 1486, 1-17. [CrossRef]

12. Violante, S.; Ijlst, L.; Te Brinke, H.; Koster, J.; de Almeida, I.T.; Wanders, R.J.A.; Ventura, F.V.; Houten, S.M. Peroxisomes contribute to the acylcarnitine production when the carnitine shuttle is deficient. Biochim. Et Biophys. Acta (BBA) Mol. Cell Biol. Lipids 2013, 1831, 1467-1474. [CrossRef] [PubMed]

13. Koves, T.R.; Ussher, J.R.; Noland, R.C.; Slentz, D.; Mosedale, M.; Ilkayeva, O.; Bain, J.; Stevens, R.; Dyck, J.R.; Newgard, C.B.; et al. Mitochondrial overload and incomplete fatty acid oxidation contribute to skeletal muscle insulin resistance. Cell Metab. 2008, 7, 45-56. [CrossRef] [PubMed]

14. Mihalik, S.J.; Goodpaster, B.H.; Kelley, D.E.; Chace, D.H.; Vockley, J.; Toledo, F.G.S.; DeLany, J.P. Increased levels of plasma acylcarnitines in obesity and type 2 diabetes and identification of a marker of glucolipotoxicity. Obes. (Silver Spring) 2010, 18, 1695-1700. [CrossRef] [PubMed]

15. Mai, M.; Tönjes, A.; Kovacs, P.; Stumvoll, M.; Fiedler, G.M.; Leichtle, A.B. Serum Levels of Acylcarnitines Are Altered in Prediabetic Conditions. PLoS ONE 2013, 8, e82459. [CrossRef] [PubMed]

16. Huffman, K.M.; Shah, S.H.; Stevens, R.D.; Bain, J.R.; Muehlbauer, M.; Slentz, C.A.; Tanner, C.J.; Kuchibhatla, M.; Houmard, J.A.; Newgard, C.B.; et al. Relationships Between Circulating Metabolic Intermediates and Insulin Action in Overweight to Obese, Inactive Men and Women. Diabetes Care 2009, 32, 1678-1683. [CrossRef] [PubMed]

17. Hunter, W.G.; Kelly, J.P.; McGarrah, R.W.; Khouri, M.G.; Craig, D.; Haynes, C.; Ilkayeva, O.; Stevens, R.D.; Bain, J.R.; Muehlbauer, M.J.; et al. Metabolomic Profiling Identifies Novel Circulating Biomarkers of Mitochondrial Dysfunction Differentially Elevated in Heart Failure With Preserved Versus Reduced Ejection Fraction: Evidence for Shared Metabolic Impairments in Clinical Heart Failure. J. Am. Heart Assoc. 2016, 5, e003190. [CrossRef] 
18. Strand, E.; Pedersen, E.R.; Svingen, G.F.T.; Olsen, T.; Bjørndal, B.; Karlsson, T.; Dierkes, J.; Njølstad, P.R.; Mellgren, G.; Tell, G.S.; et al. Serum Acylcarnitines and Risk of Cardiovascular Death and Acute Myocardial Infarction in Patients With Stable Angina Pectoris. J. Am. Heart Assoc. 2017, 6, e003620. [CrossRef] [PubMed]

19. Ahmad, T.; Kelly, J.P.; McGarrah, R.W.; Hellkamp, A.S.; Fiuzat, M.; Testani, J.M.; Wang, T.S.; Verma, A.; Samsky, M.D.; Donahue, M.P.; et al. Prognostic Implications of Long-Chain Acylcarnitines in Heart Failure and Reversibility With Mechanical Circulatory Support. J. Am. Coll. Cardiol. 2016, 67, 291-299. [CrossRef]

20. Zhao, S.; Feng, X.-F.; Huang, T.; Luo, H.-H.; Chen, J.-X.; Zeng, J.; Gu, M.; Li, J.; Sun, X.-Y.; Sun, D.; et al. The Association Between Acylcarnitine Metabolites and Cardiovascular Disease in Chinese Patients With Type 2 Diabetes Mellitus. Front. Endocrinol. 2020, 11, 212. [CrossRef]

21. Guasch-Ferré, M.; Zheng, Y.; Ruiz-Canela, M.; Hruby, A.; Martínez-González, M.A.; Clish, C.B.; Corella, D.; Estruch, R.; Ros, E.; Fitó, M.; et al. Plasma acylcarnitines and risk of cardiovascular disease: Effect of Mediterranean diet interventions. Am. J. Clin. Nutr. 2016, 103, 1408-1416. [CrossRef] [PubMed]

22. Wang, L.; Xu, F.; Song, Z.; Han, D.; Zhang, J.; Chen, L.; Na, L. A high fat diet with a high C18:0/C16:0 ratio induced worse metabolic and transcriptomic profiles in C57BL/6 mice. Lipids Health Dis. 2020, 19, 172. [CrossRef] [PubMed]

23. Harvey, K.A.; Walker, C.L.; Xu, Z.; Whitley, P.; Pavlina, T.M.; Hise, M.; Zaloga, G.P.; Siddiqui, R.A. Oleic acid inhibits stearic acid-induced inhibition of cell growth and pro-inflammatory responses in human aortic endothelial cells. J. Lipid Res. 2010, 51, 3470-3480. [CrossRef] [PubMed]

24. Fernández-Jarne, E.; Martínez-Losa, E.; Prado-Santamaría, M.; Brugarolas-Brufau, C.; Serrano-Martínez, M.; Martínez-González, M.A. Risk of first non-fatal myocardial infarction negatively associated with olive oil consumption: A case-control study in Spain. Int. J. Epidemiol. 2002, 31, 474-480. [CrossRef]

25. Maria, A.C.; Massaro, M.; Bonfrate, C.; Siculella, L.; Maffia, M.; Nicolardi, G.; Distante, A.; Storelli, C.; De Caterina, R. Oleic Acid Inhibits Endothelial Activation. Arterioscler. Thromb. Vasc. Biol. 1999, 19, 220-228. [CrossRef]

26. Zhang, C.; Hein, T.W.; Wang, W.; Chang, C.-I.; Kuo, L. Constitutive expression of arginase in microvascular endothelial cells counteracts nitric oxide-mediated vasodilatory function. FASEB J. 2001, 15, 1264-1266. [CrossRef] [PubMed]

27. Gregor, M.F.; Hotamisligil, G.S. Inflammatory mechanisms in obesity. Annu. Rev. Immunol. 2011, 29, 415-445. [CrossRef] [PubMed]

28. Chung, J.H.; Moon, J.; Lee, Y.S.; Chung, H.K.; Lee, S.M.; Shin, M.J. Arginase inhibition restores endothelial function in diet-induced obesity. Biochem. Biophys. Res. Commun. 2014, 451, 179-183. [CrossRef] [PubMed]

29. Ito, T.; Kubo, M.; Nagaoka, K.; Funakubo, N.; Setiawan, H.; Takemoto, K.; Eguchi, E.; Fujikura, Y.; Ogino, K. Early obesity leads to increases in hepatic arginase I and related systemic changes in nitric oxide and l-arginine metabolism in mice. J. Physiol. Biochem. 2018, 74, 9-16. [CrossRef]

30. Yao, L.; Bhatta, A.; Xu, Z.; Chen, J.; Toque, H.A.; Chen, Y.; Xu, Y.; Bagi, Z.; Lucas, R.; Huo, Y.; et al. Obesity-induced vascular inflammation involves elevated arginase activity. Am. J. Physiol.-Regul. Integr. Comp. Physiol. 2017, 313, R560-R571. [CrossRef] [PubMed]

31. Durante, W.; Johnson, F.K.; Johnson, R.A. Arginase: A critical regulator of nitric oxide synthesis and vascular function. Clin. Exp. Pharmacol. Physiol. 2007, 34, 906-911. [CrossRef] [PubMed]

32. Moreno, P.R.; Fuster, V. New aspects in the pathogenesis of diabetic atherothrombosis. J. Am. Coll. Cardiol. 2004, 44, 2293-2300. [CrossRef] [PubMed]

33. Kövamees, O.; Shemyakin, A.; Checa, A.; Wheelock, C.E.; Lundberg, J.O.; Östenson, C.-G.; Pernow, J. Arginase Inhibition Improves Microvascular Endothelial Function in Patients With Type 2 Diabetes Mellitus. J. Clin. Endocrinol. Metab. 2016, 101, 3952-3958. [CrossRef] [PubMed]

34. Zhang, S.; Zhang, S.; Wang, H.; Wu, W.; Ye, Y. Arginine methylation dysfunction increased risk of acute coronary syndrome in coronary artery disease population: A case-control study. Medicine 2017, 96, e6074. [CrossRef]

35. Malik, A.; Sharma, U.; Lakshmy, R.; Narang, R.; Jagannathan, N.R. Biochemical characterization of blood plasma of coronary artery disease patients by in vitro high-resolution proton NMR spectroscopy. J. Biosci. 2015, 40, 31-39. [CrossRef] [PubMed]

36. Gibala, M.J.; Young, M.E.; Taegtmeyer, H. Anaplerosis of the citric acid cycle: Role in energy metabolism of heart and skeletal muscle. Acta Physiol. Scand. 2000, 168, 657-665. [CrossRef]

37. Würtz, P.; Havulinna, A.S.; Soininen, P.; Tynkkynen, T.; Prieto-Merino, D.; Tillin, T.; Ghorbani, A.; Artati, A.; Wang, Q.; Tiainen, M.; et al. Metabolite Profiling and Cardiovascular Event Risk. Circulation 2015, 131, 774-785. [CrossRef]

38. Ruiz-Canela, M.; Toledo, E.; Clish, C.B.; Hruby, A.; Liang, L.; Salas-Salvadó, J.; Razquin, C.; Corella, D.; Estruch, R.; Ros, E.; et al. Plasma Branched-Chain Amino Acids and Incident Cardiovascular Disease in the PREDIMED Trial. Clin. Chem. 2016, 62, 582-592. [CrossRef]

39. Bhattacharya, S.; Granger, C.B.; Craig, D.; Haynes, C.; Bain, J.; Stevens, R.D.; Hauser, E.R.; Newgard, C.B.; Kraus, W.E.; Newby, L.K.; et al. Validation of the association between a branched chain amino acid metabolite profile and extremes of coronary artery disease in patients referred for cardiac catheterization. Atherosclerosis 2014, 232, 191-196. [CrossRef]

40. Tobias, D.K.; Lawler, P.R.; Harada, P.H.; Demler, O.V.; Ridker, P.M.; Manson, J.E.; Cheng, S.; Mora, S. Circulating Branched-Chain Amino Acids and Incident Cardiovascular Disease in a Prospective Cohort of US Women. Circ. Genom. Precis. Med. 2018, 11, e002157. [CrossRef] 
41. Zhang, F.; Zhao, S.; Yan, W.; Xia, Y.; Chen, X.; Wang, W.; Zhang, J.; Gao, C.; Peng, C.; Yan, F.; et al. Branched Chain Amino Acids Cause Liver Injury in Obese/Diabetic Mice by Promoting Adipocyte Lipolysis and Inhibiting Hepatic Autophagy. EBioMedicine 2016, 13, 157-167. [CrossRef] [PubMed]

42. Lynch, C.J.; Adams, S.H. Branched-chain amino acids in metabolic signalling and insulin resistance. Nat. Rev. Endocrinol. 2014, 10, 723-736. [CrossRef] [PubMed]

43. Palazoglu, M.; Fiehn, O. Metabolite Identification in Blood Plasma Using GC/MS and the Agilent Fiehn GC/MS Metabolomics RTL Library. Agil. Appl. Note 2009, 97-109.

44. Borgogna, J.-L.C.; Shardell, M.D.; Yeoman, C.J.; Ghanem, K.G.; Kadriu, H.; Ulanov, A.V.; Gaydos, C.A.; Hardick, J.; Robinson, C.K.; Bavoil, P.M.; et al. The association of Chlamydia trachomatis and Mycoplasma genitalium infection with the vaginal metabolome. Sci. Rep. 2020, 10, 3420. [CrossRef] 